\title{
Oxidative stress could precede endothelial dysfunction and insulin resistance in Indian Mauritians with impaired glucose metabolism
}

\author{
N. K. Gopaul ${ }^{1}$, M.D.Manraj ${ }^{2}$, A.Hébé ${ }^{2}$, S. Lee Kwai Yan $^{2}$, A.Johnston ${ }^{3}$, M.J. Carrier ${ }^{1}$, E.E. Änggård ${ }^{1}$ \\ ${ }^{1}$ Department of Experimental Therapeutics, William Harvey Research Institute, St. Bartholomew and the Royal London School \\ of Medicine and Dentistry, Queen Mary, London, UK \\ ${ }^{2}$ SSR Centre for Medical Studies and Research, University of Mauritius, Moka, Mauritius \\ ${ }^{3}$ Clinical Pharmacology, St. Bartholomew and the Royal London School of Medicine and Dentistry, Queen Mary, London, UK
}

\section{Abstract}

Aims/hypothesis. To measure oxidative stress, endothelial dysfunction and insulin resistance in Indian Mauritians at different stages of development of Type II (non-insulin-dependent) diabetes mellitus. Methods. Plasma total 8-epi-PGF ${ }_{2 \alpha}$, an indicator of oxidative stress, was determined in age-matched subjects with normal glucose metabolism $(n=39)$, impaired glucose tolerance $(n=14)$, newly diagnosed diabetes $(n=8)$ and established diabetes $(n=14)$. Plasma glucose and insulin were measured at baseline and $2 \mathrm{~h}$ following an oral glucose tolerance test. Endothelial function was assessed by non-invasive digital pulse wave photoplethysmography.

Results. Plasma 8-epi-PGF F $_{2 \alpha}$ increased in subjects with impaired glucose tolerance $(p<0.05)$ compared with control subjects, and was even higher in newly diagnosed diabetic patients $(p<0.01)$ and established $(p<0.01)$ diabetic patients. A tendency towards re- duced endothelial function in subjects with impaired glucose tolerance became significant in patients with newly diagnosed and established diabetes $(p<0.01)$, and was correlated with 8 -epi-PGF ${ }_{2 \alpha}(r=0.36$, $p<0.01$ ). Insulin resistance (homeostasis model assessment) did not change in subjects with impaired glucose tolerance compared with control subjects, but increased in newly diagnosed $(p<0.01)$ and established $(p<0.001)$ diabetic subjects. The 8-epi$\mathrm{PGF}_{2 \alpha}$ was correlated with fasting glucose $(r=0.50$, $p<0.001)$, triglycerides $(r=0.40, p<0.001)$ and insulin resistance $(r=0.35, p<0.001)$.

Conclusion/interpretation. Oxidant stress is an early event in the evolution of Type II diabetes and could precede the development of endothelial dysfunction and insulin resistance. [Diabetologia (2001) 44: 706712

Keywords Type II diabetes, $\mathrm{F}_{2}$-isoprostanes, oxidative stress, endothelial dysfunction, insulin resistance
Received: 1 November 2001 and in revised form: 14 February 2001

Corresponding author: N.K. Gopaul, Department of Experimental Therapeutics, William Harvey Research Institute, St. Bartholomew and Royal London School of Medicine and Dentistry, Queen Mary, Charterhouse Square, London EC1M 6BQ, UK

Abbreviations: $\mathrm{ABTS}^{+}$, 2,2'-azino-bis(3-ethylbenzothiazoline6-sulphonic acid) radical; DM, established Type II diabetes; DM-O, newly diagnosed Type II diabetes; DVP, digital volume pulse; $\mathrm{F}_{2}$-IPs, $\mathrm{F}_{2}$-isoprostanes; GC-MS, gas chromatography mass spectrometry; GTN, glyceryltrinitrate; $\mathrm{NO}^{\circ}$, nitric oxide; $\mathrm{O}_{2}{ }^{--}$, superoxide anion; $\mathrm{RI}$, reflection index; TAOS, plasma total antioxidant status.
Type II (non-insulin-dependent) diabetes mellitus is characterised by insulin resistance, pancreatic betacell defect, hypertension, dyslipidaemia and central obesity, and is recognised as part of a cluster of cardiovascular risk factors generally known as the Metabolic Syndrome [1]. The Indian Ocean island of Mauritius recently provided the background for a longitudinal epidemiological study (1987-1998) which is now seen as an indicator of the potential global impact of Type II diabetes [2,3]. Mauritius has a multiethnic population of 1.2 million, including people of Asian Indian $(66 \%)$, mixed European-African-Indian $(31 \%)$ and Chinese (3\%) descent. In 1987, the agestandardised prevalence of Type II diabetes in adults aged 30 years or more was $14.3 \%$, increasing to 
$16.9 \%$ in 1992 and $19.5 \%$ in 1998 [4]. Current data indicate an age-adjusted prevalence of impaired glucose tolerance (IGT) in Mauritian men and women (age range 30-64 years) of $9.6-17.1 \%$ and $19.7-21.9 \%$, respectively [5].

The aim of this study was to examine the relation between oxidative stress, endothelial dysfunction and insulin resistance in Mauritian subjects of Asian Indian descent with varying levels of impaired glucose metabolism, representing different stages in the development of Type II diabetes. We have proposed that oxidative stress precedes endothelial dysfunction and the subsequent development of insulin resistance $[6,7]$. Oxidative stress was assessed by measurement of plasma $\mathrm{F}_{2}$-isoprostanes $\left(\mathrm{F}_{2}\right.$-IPs $)$, which are prostaglandin-like molecules produced by the free radical-catalysed peroxidation of arachidonic acid [8]. Plasma and urinary concentrations of one of the abundant $\mathrm{F}_{2}$-IPs, 8-epi-PGF $2 \alpha$, are higher in subjects with Type II diabetes than in healthy control subjects [9] and decrease following dietary supplementation with antioxidants $[10,11]$. Similar observations have been reported in animal models of diabetes [12,13] and it is now recognised that measurement of 8-epi$\mathrm{PGF}_{2 \alpha}$ represents a sensitive and reliable index of oxidative stress in vivo [14].

Endothelial dysfunction has been reported in patients with established Type II diabetes [15-20] and in elderly subjects with impaired fasting glucose (IFG) [21], suggesting that even a moderate disturbance in glucose metabolism could constitute a significant risk for diabetic endotheliopathy. In this study, we assessed endothelial dysfunction using non-invasive digital photoplethysmography in combination with endothelium-independent and endothelium-dependent vasodilators [22].

\section{Subjects and methods}

Subjects. Recruitment of subjects for this study was carried out at the SSR Centre for Medical Studies and Research in Mauritius. Subjects of North-Indian descent were selected from the population over a 3-month period. The majority of subjects participating in this study visited the centre for the first time but a few subjects who are involved in ongoing family studies at the SSR Centre were also included. All subjects in this study underwent a clinical examination and a resting electrocardiogram (ECG). Those with clinical evidence of ischaemic heart disease were excluded. Subjects with hypertension (systolic blood pressure $\geq 140 \mathrm{~mm} \mathrm{Hg}$ or diastolic blood pressure $\geq 90 \mathrm{~mm} \mathrm{Hg}$ ), high total or low density lipoprotein cholesterol ( $\geq 7$ and/or $>5 \mathrm{mmol} / \mathrm{l}$, respectively) and smokers were also excluded. Diabetes was diagnosed using the World Health Organisation (WHO) criteria [23]. Subjects with diabetes were not treated with either hypoglycaemic agents or insulin. None of the subjects were taking antioxidant vitamin supplements, lipid-lowering drugs or beta blockers. This study was approved by the Ethics and Scientific Advisory committees of the University of Mauritius. All participants provided written, informed consent.
Blood sampling and plasma preparation. Participants were invited to attend the SSR Centre after an overnight fast. Following registration, fasting blood samples were collected from all the subjects. To determinate of 8 -epi-PGF $2 \alpha$, blood $(10 \mathrm{ml})$ was collected from the brachial vein in polyethylene tubes containing a $3.8 \%(\mathrm{w} / \mathrm{v})$ trisodium citrate solution (blood/anticoagulant ratio of 9/1), indomethacin (as cyclooxygenase inhibitor) and butylated hydroxytoluene (BHT, as free radical scavenger) at final concentrations of 14 and $20 \mu \mathrm{mol} / \mathrm{l}$, respectively. The sample was allowed to stand for $30 \mathrm{~min}$ at $4{ }^{\circ} \mathrm{C}$ to enable complete inhibition of cyclooxygenase enzymes. Platelet-poor plasma was obtained by centrifugation at $1120 \mathrm{~g}$ for $15 \mathrm{~min}$ at $4{ }^{\circ} \mathrm{C}$. The was transferred to a polypropylene screw-cap tube and BHT added at a final concentration of $20 \mu \mathrm{mol} / 1$. The sample was then stored at $-80^{\circ} \mathrm{C}$ until analysis. For the determination of plasma triglycerides, cholesterol, uric acid, urea and creatinine, fasting blood was collected in heparin tubes. For the measurement of plasma insulin, total antioxidant status (TAOS) and vitamin E, blood was collected in EDTA tubes. After centrifugation, plasma was transferred to screw-cap polypropylene tubes and stored at $-80^{\circ} \mathrm{C}$ until analysis. A second blood sample was taken $2 \mathrm{~h}$ following an oral glucose tolerance test (OGTT, $75 \mathrm{~g}$ anhydrous glucose in $250 \mathrm{ml}$ water) for measurement of TAOS, insulin and glucose. For glucose determinations, fasting and 2-h blood samples were collected into tubes containing potassium fluoride. Following centrifugation, plasma glucose was assayed by midday at the latest after collection of the last 2-h sample. All plasma samples for the measurement of 8 -epi-PGF $2 \alpha$, TAOS and insulin were stored at $-80^{\circ} \mathrm{C}$ and were sent to the William Harvey Research Institute by air on dry ice in two separate shipments. On arrival, samples were stored at $-80^{\circ} \mathrm{C}$ until analysis.

Additional subject information. As arranged when booking their appointment, participants were asked to provide a midstream early morning urine sample for the measurement of albuminuria and microalbuminuria. Prior to administration of the OGTT, anthropometric measurements were carried out with the subjects wearing light clothes and without footwear.

Analysis of 8-epi-PGF $F_{2 \alpha}$. Plasma samples were subjected to alkaline hydrolysis for the measurement of total (sum of free plus esterified) 8-epi-PGF $2 \alpha$ by gas chromatography - mass spectrometry (GC-MS), as described previously [24]. The GC-MS assay has a limit of detection of about $10 \mathrm{pg} / \mathrm{ml}(28$ $\mathrm{pmol} / \mathrm{l}$ ) in plasma with intra-assay and inter-assay coefficients of variation of 4.4 and $7.6 \%$, respectively.

Plasma total antioxidant status measurement. The total antioxidant status of plasma was determined by its capacity to inhibit the peroxidase-mediated formation of the 2,2'-azino-bis(3-ethylbenzothiazoline-6-sulphonic acid) radical $\left(\mathrm{ABTS}^{+}\right)$, as described earlier [24]. The intra-assay and inter-assay coefficients of variation are less than 2 and less than $5 \%$, respectively.

Additional measurements. Plasma lipid hydroperoxides were measured by spectrophotometry at $560 \mathrm{~nm}$ using the ferrous oxidation-xylenol orange (FOX-2) assay [25]. Plasma vitamin $\mathrm{E}(\alpha-$ and $\gamma$-tocopherol) was measured by reversed-phase high-performance liquid chromatography and fluorescence detection, as described previously [24]. Plasma glucose, triglycerides, total cholesterol, HDL-cholesterol and uric acid were determined by enzymatic colorimetric methods using a multiparametric Cobas Mira Plus auto-analyser (Roche Diagnostics Systems, Produits Roche, Neuilly-sur-Seine, France). Urea was assayed using a UV enzymatic kinetic method and creatinine by a colorimetric endpoint method. Assay reagent kits, 
standards (multiparametric calibrator human serum) and control samples (two multiparametric assayed human control sera, one normal and one elevated) were obtained from Randox (Randox Laboratories, Antrim, UK). LDL cholesterol was calculated using the Friedewald formula [26]. Plasma insulin was measured using a double-antibody radioimmunoassay (Linco Research, St. Louis, Miss., USA). An estimate of insulin sensitivity was derived by homeostasis model assessment (HOMA) using the formula: fasting plasma glucose $(\mathrm{mmol} / \mathrm{l})$ $\mathrm{X}$ fasting plasma insulin $(\mu \mathrm{U} / \mathrm{ml}) / 22.5$, as described previously [27]. Blood haemoglobin was determined by a colorimetric endpoint method.

Endothelial function assessment. Endothelial function was assessed in a subset of participants, using a portable photoplethysmograph (Micro Medical MP2000, Gillingham, Kent, UK) as described earlier [22]. Digital volume pulse (DVP) data were collected using a probe placed on the index finger of the right hand and in-built software was applied to provide a reflection index (RI) related to tone in small arteries. The reflection index was calculated as the height of the inflection point after the first peak of the DVP, and expressed as a percentage height of the first peak (Fig. 1).

Data collection was carried out with the subject supine in a temperature-controlled laboratory $\left(27 \pm 1^{\circ} \mathrm{C}\right)$. Each subject was allowed to acclimatise to the laboratory temperature for $30 \mathrm{~min}$ before the start of measurements. Baseline recordings were carried out at $5 \mathrm{~min}$ intervals for a total of $15 \mathrm{~min}$ and a mean baseline RI was calculated ( $\left.\mathrm{RI}_{\text {BASELINE }}\right)$. Endothelial responses were then measured following administration of an endothelium-independent vasodilator (glyceryltrinitrate (GTN), $500 \mu \mathrm{g}$ sublingual for $3 \mathrm{~min}$ ) and an endothelium-dependent vasodilator (salbutamol, $400 \mu \mathrm{g}$ by inhalation through a spacer). Each recording was carried out for $30 \mathrm{~s}$ and calculations based on the final 3 waveforms. In the case of GTN, the mean of measurements at 3 min was used to calculate the endothelial response $\left(\mathrm{RI}_{\mathrm{GTN}}\right)$. The response to salbutamol was taken as the mean of measurements at 10 and 15 min following inhalation ( $\left.\mathrm{RI}_{\text {SALBUTAMOL }}\right)$. The change in RI from baseline following GTN and salbutamol was expressed as $\left(\mathrm{RI}_{\mathrm{GTN}}\right.$ minus $\mathrm{RI}_{\mathrm{BASE}}$ LINE) and ( $\mathrm{RI}_{\text {SALBUTAMOL }}$ minus $\left.\mathrm{RI}_{\text {BASELINE }}\right)$, respectively. In situations where endothelial responses to salbutamol are normal, $\mathrm{RI}_{\text {SALBUTAMOL }}$ is less than $\mathrm{RI}_{\text {BASELINE }}$, resulting in a negative change in RI. In cases where endothelial responses are blunted, $\mathrm{RI}_{\text {SALBUTAMOL }}$ is greater than $\mathrm{RI}_{\text {BASELINE }}$ and results in a positive change in RI. Blood pressure and pulse were recorded every $5 \mathrm{~min}$ throughout the test. The intra-individual coefficient of variation of RI using this approach ranges from $4.4-6.4 \%$.

Statistical analysis. Data are expressed as means \pm SEM, or as median and range where the distribution is skewed. Comparison between mean values in groups with normally distributed data was done by parametric one-way analysis of variance (ANOVA) followed by Dunnett's test, using the subjects with normal glucose tolerance as control. Non-parametric (Kruskal-Wallis) ANOVA followed by Dunn's test was applied for data with skewed distribution. A $p$ value of less than 0.05 was considered statistically significant. Associations between the different variables were examined by determination of Pearson's correlation coefficient. Statistical calculations were carried out using Minitab Statistical Software, version 13.20 (Minitab, PA., USA).

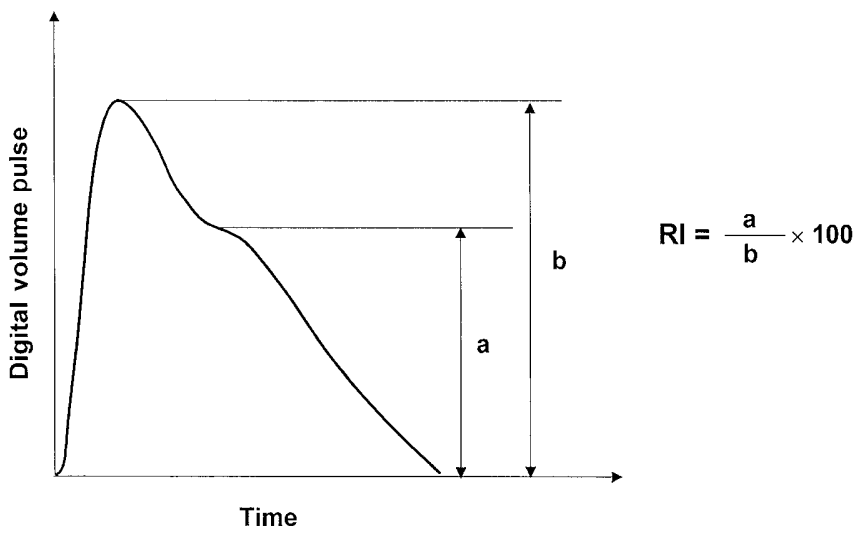

Fig. 1. The digital volume pulse (DVP) waveform. The DVP exhibits a characteristic 'notch' or inflection point in its waveform, which changes following administration of endothelium-dependent or endothelium-independent vasodilators. The amplitude of this inflection point, a, can be expressed as a percentage of the maximal DVP amplitude, $\mathbf{b}$, and is termed the reflection index $(\mathrm{RI})$

\section{Results}

A total of 75 subjects ( 36 men and 39 women) were studied, grouped according to those with normal glucose tolerance (control, $n=39$ ), impaired glucose tolerance (IGT, $n=14$ ), newly diagnosed diabetes (DM$\mathrm{O}, n=8)$ and established diabetes (DM, $n=14)(\mathrm{Ta}-$ ble 1).

The male:female ratio in each of the groups was different. In the case of the DM-O group, 6 subjects diagnosed with Type II diabetes had no previous record of an OGTT and were not aware of their impaired glucose metabolism prior to participating in this study. The remaining 2 subjects in this group had been classified as IGT at their previous OGTT, approximately 2 years earlier. Duration of diagnosed diabetes in the DM group ranged from 2-8 years. Fasting plasma insulin concentrations were highest in the DM-O group, representing a 1.7-fold increase $(p<0.001)$ compared to control subjects. In the DM group, fasting insulin concentrations were 1.5 -fold higher compared to control subjects $(p<0.05)$. Insulin resistance determined by HOMA was increased in the DM-O $(p<0.01)$ and DM $(p<0.001)$ groups, compared to control subjects (Fig. 2A).

Post-OGTT insulin concentrations in the control, IGT, DM-O and DM groups were 6.7-, 11.3-, 9.2and 5.3-fold higher, respectively, than the corresponding fasting concentrations. Plasma triglycerides were higher in subjects with established diabetes $(p<0.01)$ in comparison to control subjects. Although total cholesterol was similar among the different subject groups, LDL cholesterol was higher in DM-O $(p<0.01)$ and HDL cholesterol lower in IGT and DM $(p<0.05)$ groups, in comparison to the corresponding controls. 
Table 1. Physical and biochemical characteristics of subjects in the study groups

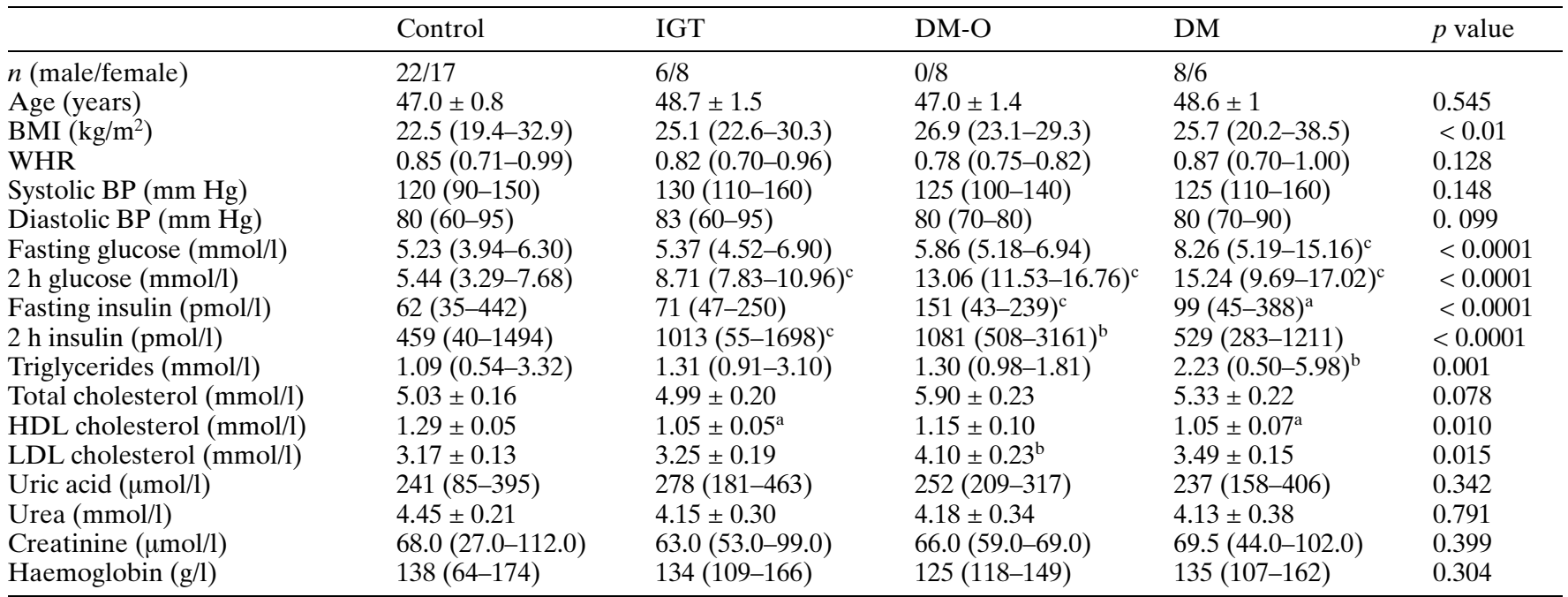

Data are expressed as means \pm SEM or as median (range) for skewed data. Differences between group means were tested by oneway ANOVA.

${ }^{\mathrm{a}} p<0.05 ;{ }^{\mathrm{b}} p<0.01 ;{ }^{\mathrm{c}} p<0.001$ vs control subjects

Table 2. Influence of impaired glucose metabolism on plasma markers of oxidative stress

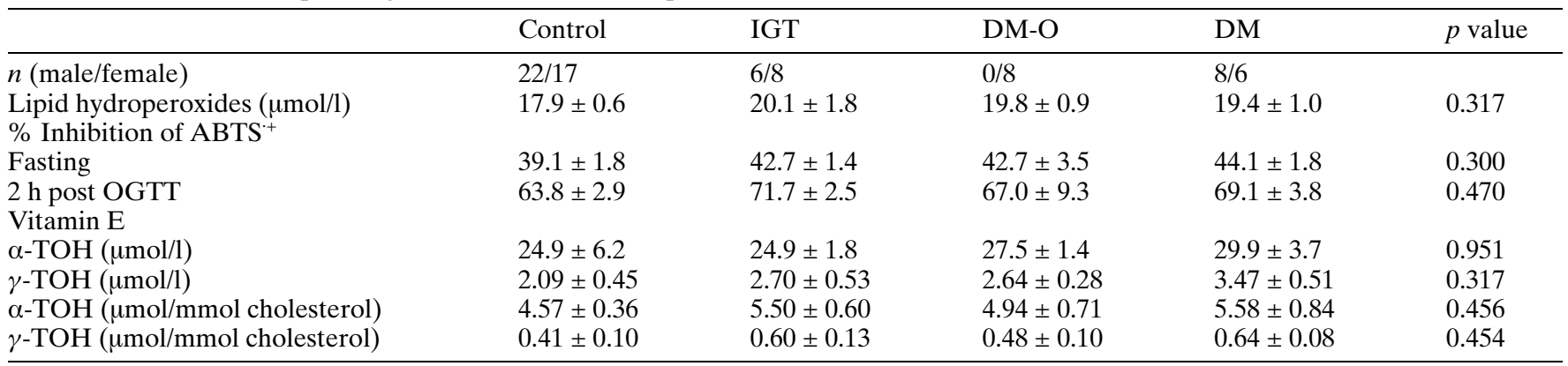

Data are expressed as means \pm SEM. Differences between group means were tested by one-way ANOVA

Plasma concentrations of total 8-epi- $\mathrm{PGF}_{2 \alpha}$ were higher in the IGT $(p<0.05)$, DM-O $(p<0.01)$ and DM $(p<0.01)$ groups compared to control groups (Fig. 2B). Multiple correlations with fasting total 8epi-PGF ${ }_{2 \alpha}$ as the dependent variable revealed a positive correlation with fasting glucose $(r=0.50$, $p<0.001)$, triglycerides $(r=0.40, p<0.001)$, insulin resistance (HOMA) $(r=0.35, p<0.001)$ and TAOS $(r=0.23, p<0.05)$. When the relation of 8 -epi$\mathrm{PGF}_{2 \alpha}$ to the fasting variables was analysed using stepwise multiple regression, only fasting glucose $(p<0.001)$ and triglycerides $(p<0.05)$ were independently significant. No significant differences between the subject groups were observed in lipid hydroperoxides, fasting and 2-h plasma TAOS, or vitamin E ( $\alpha$ - and $\gamma$ - tocopherol) (Table 2).

Interestingly, post-OGTT plasma TAOS increased approximately 1.6-fold in all subject groups following this test, compared to the corresponding fasting TAOS. To exclude a possible influence of glucose on the measurement of TAOS, glucose solutions of 5 ,
10 and $15 \mathrm{mmol} / \mathrm{l}$ were added directly to the assay. In addition, six control plasma samples were tested before and after addition of $15 \mathrm{mmol} / \mathrm{l}$ (final concentration) glucose. In all these cases, glucose did not interfere with the determination of plasma TAOS.

Endothelial dysfunction was assessed in a subset of the participants from the control group $(n=25)$, IGT $(n=8)$, DM-O $(n=6)$ and DM $(n=9)$ groups. Changes in RI response were determined following GTN and salbutamol administration. Baseline RI values in control subjects were similar to those observed in the other groups. At the doses used in this study, GTN resulted in a larger change in RI response than that produced by salbutamol $(p<0.001)$, although no significant differences were observed between the subject groups. The RI response following salbutamol was blunted in DM-O and DM compared to the control group $(p<0.01)$ (Fig. 2C). Finally, when the relation of 8 -epi-PGF ${ }_{2 \alpha}$ to endothelial function and fasting biochemical parameters was analysed using stepwise multiple regression analysis, only fasting 

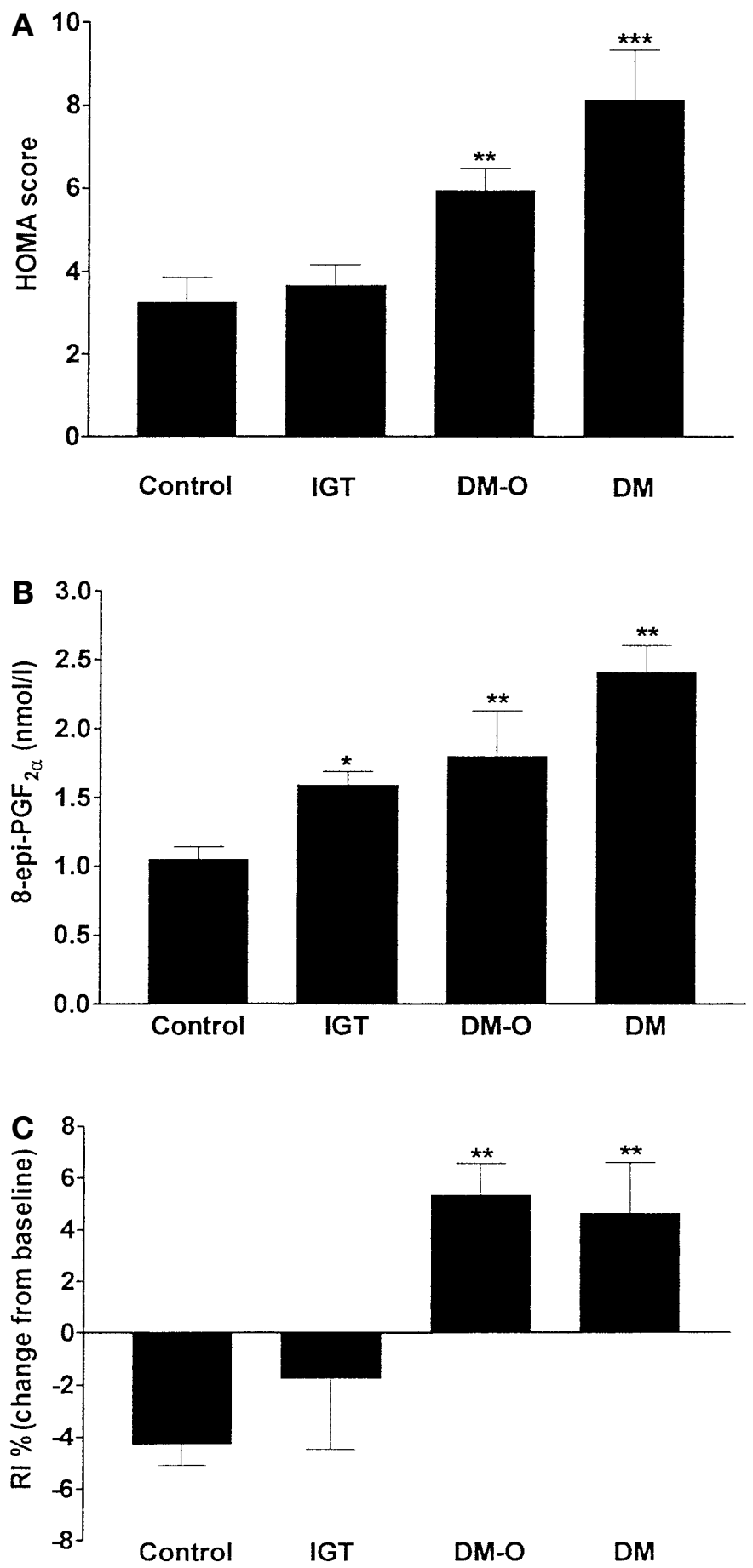

Fig. 2 A-C. Measurement of insulin resistance $(\mathbf{A})$, oxidative stress (B) and endothelium-dependent endothelial response (C) in subjects with varying levels of impaired glucose metabolism. Insulin resistance (HOMA) and oxidative stress (plasma total 8-epi-PGF ${ }_{2 \alpha}$ measured by immunoaffinity extraction and GC-MS) were determined in control subjects $(n=39)$, and in those with IGT $(n=14)$, newly diagnosed diabetes (DM-O, $n=8)$ and established diabetes (DM, $n=14)$. Endothelial responses, expressed as a reflection index (RI) related to tone in small arteries, were measured by digital photoplethysmography following the administration of salbutamol $(400 \mu \mathrm{g}$ by inhalation) in control $(n=25)$, IGT $(n=8)$, DM-O $(n=6)$ and $\mathrm{DM}(n=9)$ subjects. Data are expressed as means \pm SEM. Differences between group means were tested by one-way ANOVA. $* p<0.05 ; * * p<0.01 ; * * * p<0.001$ vs control subjects glucose $(p<0.01)$ and RI responses to salbutamol $(p<0.01)$ were independently significant.

\section{Discussion}

In this study we have shown that increased oxidative stress and endothelial dysfunction are associated with the development of Type II diabetes in Mauritians of Asian Indian descent. Circulating concentrations of 8-epi-PGF ${ }_{2 \alpha}$ (currently regarded as the most reliable index for the assessment of lipid peroxidation in humans) in the IGT, DM-O and DM groups were 1.5-fold, 1.7-fold and 2.3-fold higher, respectively, compared to the control group, indicating increasing oxidative stress with progressive dysglycaemia. A significant correlation between 8 -epi-PGF ${ }_{2 \alpha}$ and fasting glucose suggests an association between glycaemia and increased lipid peroxidation among the subjects in this study. Several potential mechanisms could account for the increase in 8-epi-PGF ${ }_{2 \alpha}$ formation, including glucose autoxidation [28] and advanced glycation end products (AGE) formation [29]. Furthermore, a relatively small amount of 8 -epi-PGF ${ }_{2 \alpha}$ could be formed using a cyclooxygenase-dependent pathway [30]. 8-epi-PGF ${ }_{2 \alpha}$ is a potent vasoconstrictor and is involved in the activation of intracellular signalling, suggesting that it could act as an important mediator in the manifestation of endotheliopathy in the early stages of Type II diabetes, either directly, or through the stimulated synthesis of other vasoconstrictor agents such as endothelin-1 [31,32].

The absence of significant changes in plasma TAOS and vitamin E between the groups in our study is in agreement with a recent report [33], where no significant differences were found in plasma total antioxidant activity and vitamin $\mathrm{E}$ in both Caucasian and Indo-Asian subjects with Type II diabetes, compared to corresponding control subjects matched for age. It is conceivable, however, that small but significant changes in individual antioxidants were not detected in our study. In contrast to a recent report, where decreased antioxidant defences were found during the OGTT [34], we observed a $35-38 \%$ increase in the 2-h plasma TAOS, suggesting that the adaptive response of antioxidant enzymes to the short term hyperglycaemia-induced rise in oxidant stress was preserved [35].

Our data show a blunting of the endothelium-dependent response following salbutamol administration, in subjects with Type II diabetes (DM-O and DM groups). This is significantly correlated with plasma 8-epi-PGF ${ }_{2 \alpha}$ and insulin sensitivity (HOMA) and is in agreement with an earlier report [22] suggesting, in part, an impairment in the endothelial L-arginine nitric oxide (NO) pathway. Apart from the reduced formation of $\mathrm{NO}$, several mechanisms including impaired transport of the released $\mathrm{NO}^{\circ}$ and the simulta- 
neous formation of vasoconstrictor mediators, could also account for the impaired endothelium-dependent responses observed in diabetic subjects. Indeed, the higher 8-epi-PGF ${ }_{2 \alpha}$ concentrations in subjects with Type II diabetes could reflect a reduced bioavailability of $\mathrm{NO}$ due to excessive production of $\mathrm{O}_{2}{ }^{--}$. The combination of $\mathrm{O}_{2}^{-{ }^{-}}$and $\mathrm{NO}^{\circ}$ can potentially generate peroxynitrite and hydroxyl radicals, which could further aggravate endothelial dysfunction. The high plasma triglyceride concentrations in the DM group are consistent with a reduction in endothelial lipoprotein lipase activity and an increased flux of free fatty acids, and could contribute to endothelial dysfunction [36]. Interestingly, although impaired endothelial function was found in DM-O subjects, triglyceride concentrations were not significantly different compared to control subjects whereas LDL cholesterol concentrations were significantly higher. Higher LDL cholesterol can decrease the activity of endothelial nitric oxide synthase (eNOS) by promoting the inhibitory interaction between eNOS and caveolin, resulting in decreased NO formation [37]. There was a trend towards decreased endotheliumdependent responses in subjects with IGT compared to control subjects but the difference was not significant and could be related to an early compensatory mechanism in the presence of reduced NO availability [38].

The HOMA model has been proposed as a useful tool for the assessment of insulin sensitivity in large studies, where it is less practical to adopt more complex procedures [39]. In this study, a positive correlation was found between the HOMA score and plasma 8 -epi-PGF ${ }_{2 \alpha}$, with lower insulin concentrations in the DM group compared to DM-O indicating progressive pancreatic beta-cell failure. Plasma 8-epi-PGF ${ }_{2 \alpha}$ derives to a large extent from LDL present in circulation and therefore reflects LDL oxidation in vivo. Recent clinical studies have shown inverse relation between vascular function and markers of LDL oxidation $[40,41]$ and suggest that elevated oxidative stress brought about by an increased proportion of oxidised LDL in circulation could contribute to the endothelial dysfunction observed in Type II diabetes. Localised excess $\mathrm{O}_{2}{ }^{--}$could conceivably contribute to the inactivation of NO without influencing the total antioxidant capacity in plasma. Consequently, endothelial dysfunction could occur without detectable changes in the hydrophilic antioxidants. Indeed, endothelial dysfunction was recently reported to be independent of plasma total radical trapping capacity (TRAP), ascorbic acid, protein-bound thiols or uric acid [21].

This study, which has shown that elevated 8-epi$\mathrm{PGF}_{2 \alpha}$ and endothelial dysfunction are associated with the development of Type II diabetes in Mauritians of Asian Indian origin, needs to be investigated further using a larger cohort. Interestingly, we have also shown that higher oxidative stress was present in the prediabetic stage (IGT), in the absence of significant changes in endothelial function and insulin sensitivity. Our data support the contention that oxidative stress could precede endothelial dysfunction and the appearance of insulin resistance, as proposed recently $[42,43]$.

Acknowledgements. The authors thank Mrs J. Adekalon for the excellent technical assistance.

\section{References}

1. Zimmet PZ (1995) The pathogenesis and prevention of diabetes in adults: Genes, autoimmunity and demography. Diabetes Care 18: 1050-1064

2. Dowse GK, Gareeboo H, Zimmet PZ et al. (1990) High prevalence of NIDDM and impaired glucose tolerance in Indian, Creole and Chinese Mauritians. Diabetes 39: 390-396

3. Zimmet P (2000) Globalization, coca-colonization and the chronic disease epidemic: can the Doomsday scenario be averted? J Intern Med 247: 301-310

4. De Courten M, Chitson P, Cox HS, Alberti KGMM, Tuomilehto J, Zimmet PZ (1999) The rise and rise of diabetes in Mauritius 1987-1998. Diabetes 48 [Suppl 1] A1758 (Abstract)

5. World Health Organisation (WHO) diabetes database (2000) Prevalence of impaired glucose tolerance (\% population) in the age range $30-64$ years in African and Asian study populations. WHO web site www.who.int/ncd/dia/databases2.htm\#t1

6. Laight DW, Carrier MJ, Änggård EE (1999) Endothelial cell dysfunction and the pathogenesis of diabetic macroangiopathy. Diabetes Metab Res Rev 15: 274-282

7. Laight DW, Carrier MJ, Änggård EE (2000) Antioxidants, diabetes and endothelial dysfunction. Cardiovasc Res 47: 457-464

8. Morrow JD, Hill KE, Burk RE, Nammour TM, Badr KF, Roberts LJ 2nd (1990) A series of prostaglandin $\mathrm{F}_{2}$-like compounds are produced in vivo in humans by a non-cyclooxygenase, free radical-catalysed mechanism. Proc Natl Acad Sci USA 87: 9383-9387

9. Gopaul NK, Änggård EE, Mallet AI, Betteridge DJ, Wolff SP, Nourooz-Zadeh J (1995) Plasma 8-epi-PGF $2 \alpha$ levels are elevated in individuals with non-insulin dependent diabetes mellitus. FEBS Lett 368: 225-229

10. Davi G, Ciabattoni G, Consoli A et al. (1999) In vivo formation of 8-epi-prostaglandin $\mathrm{F}_{2 \alpha}$ and platelet activation in diabetes mellitus: Effects of improved metabolic control and vitamin E supplementation. Circulation 99: 224-229

11. Chowienczyk PJ, Brett SE, Gopaul NK et al. (2000) Oral treatment with an antioxidant (raxofelast) reduces oxidative stress and improves endothelial function in men with Type II diabetes. Diabetologia 43: 974-977

12. Laight DW, Desai KM, Gopaul NK, Änggård EE, Carrier MJ (1999) Pro-oxidant challenge in vivo provokes the onset of NIDDM in the insulin resistant obese Zucker rat. Br J Pharmacol 128: 269-271

13. Palmer AM, Thomas CR, Gopaul N et al. (1998) Dietary antioxidant supplementation reduces lipid peroxidation but impairs vascular function in small mesenteric arteries of the streptozotocin-diabetic rat. Diabetologia 41: 148-156 
14. Halliwell B (2000) Lipid peroxidation, antioxidants and cardiovascular disease: how should we move forward? Cardiovasc Res 47: 410-418

15. McVeigh GE, Brennan GM, Johnston GD et al. (1992) Impaired endothelium-dependent and independent vasodilatation in patients with Type II (non-insulin-dependent) diabetes mellitus. Diabetologia 35: 771-776

16. Goodfellow J, Ramsey MW, Luddington LA et al. (1996) Endothelium and inelastic arteries: an early marker of vascular dysfunction in non-insulin-dependent diabetes. BMJ 312: 744-745

17. Ting HH, Timimi K, Boles K, Creager SJ, Ganz P, Creager MA (1996) Vitamin C improves endothelium-dependent vasodilatation in patients with non-insulin-dependent diabetes mellitus. J Clin Invest 97: 22-28

18. Watts GF, O'Brien SF, Silvester W, Millar JA (1996) Impaired endothelium-dependent and independent dilatation of forearm resistance arteries in men with diet-treated noninsulin-dependent diabetes: role of dyslipidaemia. Clin Sci (Colch) 91: 567-573

19. Williams SB, Cusco JA, Roddy M-A, Johnstone MT, Creager MA (1996) Impaired nitric oxide-mediated vasodilatation in patients with non-insulin-dependent diabetes mellitus. J Am Coll Cardiol 27: 567-574

20. Mäkimattila S, Ming-Lin L, Vakkilainen J et al. (1999) Impaired endothelium-dependent vasodilatation in NIDDM: relation to LDL size, oxidized LDL and antioxidants. Diabetes Care 22: 973-981

21. Vehkavaara S, Seppälä-Lindroos A, Westerbacka J, Groop PH, Yki-Järvinen H (1999) In vivo endothelial dysfunction characterizes patients with impaired fasting glucose. Diabetes Care 22: 2055-2060

22. Chowienczyk PJ, Kelly RP, McCallum H et al. (1999) Photoplethysmographic assessment of pulse wave reflection. Blunted response to endothelium-dependent beta $a_{2}$-adrenergic vasodilation in type II diabetes mellitus. J Am Coll Cardiol 34: 2007-2014

23. World Health Organisation Study Group on Diabetes Mellitus (1985) Technical report series No 727, WHO, Geneva

24. Gopaul NK, Zacharowski K, Halliwell B, Änggård EE (2000) Evaluation of the postprandial effects of a fast-food meal on human plasma $\mathrm{F}_{2}$-isoprostane levels. Free Radic Biol Med 28: 806-814

25. Jiang ZY, Hunt JV, Wolff SP (1992) Ferrous ion oxidation in the presence of xylenol orange for detection of lipid hydroperoxides in low density lipoprotein. Anal Biochem 202: 384-389

26. Friedewald WT, Levy RI, Frederikson DS (1972) Estimation of the concentration of low-density lipoprotein cholesterol in plasma without the use of the preparative centrifuge. Clin Chem 18: 499-502

27. Matthews D, Hosker J, Rudenski A, Naylor B, Treacher D, Turner R (1985) Homeostasis model assessment: insulin resistance and $\beta$-cell function from fasting plasma glucose and insulin concentrations in man. Diabetologia 28: $412-419$
28. Hunt JV, Smith CCT, Wolff SP (1990) Autoxidative glycosylation and possible involvement of peroxides and free radicals in LDL modification by glucose. Diabetes 39: 1420-1424

29. Schmidt AM, Yan SD, Wautier JL, Stern D (1999) Activation of receptor for advanced glycation end products: a mechanism for chronic vascular dysfunction in diabetic vasculopathy and atherosclerosis. Circ Res 84: 489-497

30. Pratico D, Lawson JA, FitzGerald GA (1995) Cyclooxygenase-dependent formation of the isoprostane, 8-epi prostaglandin $\mathrm{F}_{2 \mathrm{a}}$. J Biol Chem 270: 9800-9808

31. Fukunaga M, Yura T, Badr KF (1995) Stimulatory effect of 8-epi-PGF $\mathrm{Pa}_{2 \mathrm{a}}$, an $\mathrm{F}_{2}$-isoprostane, on endothelin-1 release. J Cardiovasc Pharmacol 26 [Suppl.]: S51-S52

32. Seligman BGS, Biolo A, Polanczyk CA, Gross JL, Clausell N (2000) Increased plasma levels of endothelin 1 and von Willebrand Factor in patients with Type 2 diabetes and dyslipidemia. Diabetes Care 23: 1395-1400

33. Wen Y, Sahni A, Rea CA, Zhang X-H, Khokher MA, Singh BM (2000) Differential antioxidant status among Indo-Asians compared with Caucasians with and without diabetes. Diabetes Care 23: 254-255

34. Ceriello A, Bortolotti N, Crescentini A et al. (1998) Antioxidant defences are reduced during the oral glucose tolerance test in normal and non-insulin-dependent diabetic subjects. Eur J Clin Invest 28: 329-333

35. Ceriello A, Dello Russo P, Amstad P, Cerutti P (1996) High glucose induces antioxidant enzymes in human endothelial cells in culture: evidence linking hyperglycaemia and oxidative stress. Diabetes 45: 471-477

36. Pinkney JH, Coen DA, Stehouwer CDA et al. (1997) Endothelial dysfunction: cause of the insulin resistance syndrome. Diabetes 16: S9-S13

37. Feron O, Dessy C, Moniotte S, Desager JP, Balligand JL (1999) Hypercholesterolemia decreases nitric oxide production by promoting the interaction of caveolin and endothelial nitric oxide synthase. J Clin Invest 103: 897-905

38. Taddei S, Virdis A, Ghiadoni L, Salvetti G, Salvetti A (2000) Endothelial dysfunction in hypertension. J Nephrol 13: 205-210

39. Bonora E, Targher G, Alberiche M et al. (2000) Homeostasis model assessment closely mirrors the glucose clamp technique in the assessment of insulin sensitivity: studies in subjects with various degrees of glucose tolerance and insulin sensitivity. Diabetes Care 23: 57-63

40. Anderson TJ, Meredith IT, Charbonneau F et al. (1996) Endothelium-dependent coronary vasomotion relates to the susceptibility of LDL to oxidation in humans. Circulation 93: 1647-1650

41. Raitakari OT, Pitkänen OP, Lehtimäki T et al. (1997) In vivo LDL oxidation relates to coronary reactivity in young men. J Am Coll Cardiol 30: 97-102

42. Tooke JE, Goh KL (1998) Endotheliopathy precedes Type II diabetes. Diabetes Care 21: 2047-2049

43. Wiernsperger N (2000) Defects in microvascular haemodynamics during prediabetes: contributor or epiphenomenon? Diabetologia 43: 1439-1448 